\title{
Força de Clima: Seu Papel Moderador na Relação Entre Clima e Turnover
}

\author{
Climate Strength: Its Role as a Moderator in the Relationship Between Climate and \\ Turnover
}

Tatiana Iwai ${ }^{1}$

(iD) https://orcid.org/0000-0002-8733-5369

Adriana Bruscato Bortoluzzo ${ }^{1}$

(iD) https://orcid.org/0000-0003-2872-031X

Lina Eiko Nakata ${ }^{2}$

https://orcid.org/0000-0003-3549-0663

José Eduardo Teixeira Costa ${ }^{1}$

https://orcid.org/0000-0002-1475-7456

Insper, São Paulo, SP, Brasil ${ }^{1}$ Great Place to Work, São Paulo, SP, Brasil ${ }^{2}$

Artigo recebido em 28.06.2017. Última versão recebida em 17.08.2018. Aprovado em 19.08.2018. 


\title{
Resumo
}

Na literatura, o efeito da qualidade do clima, definido como as percepções positivas sobre o ambiente de trabalho, nos resultados organizacionais já é relativamente bem conhecido e estudado. No entanto, nos últimos anos, a literatura de clima tem prestado atenção crescente em um novo construto: força de clima, que se refere ao grau de consenso entre as pessoas sobre o clima dentro da unidade de trabalho ou organização. Para avançar na investigação do papel de clima nos resultados organizacionais, o presente trabalho examina o efeito de força de clima no turnover em nível organizacional. Para isso, usou-se uma amostra composta de 25.288 indivíduos de 150 empresas de médio e grande porte de diversos setores em que se testou o efeito direto e o moderador de força de clima em cinco dimensões de clima no nível de turnover coletivo. Os resultados obtidos mostraram que, após controlar a qualidade do clima organizacional, a força de clima modera a relação entre qualidade de clima e turnover coletivo em três das cinco facetas de clima estudadas, de forma que, quando a força de clima é alta, a influência do clima organizacional no turnover é potencializada, enquanto que, quando a força de clima é baixa, essa influência se enfraquece. Porém, não há efeito direto entre força de clima e turnover coletivo.

Palavras-chave: clima organizacional; força de clima; turnover coletivo; consenso de percepções.

\begin{abstract}
The effects of climate quality, defined as the shared perceptions of the work environment, on organizational outcomes is already well known and studied in the literature. However, in recent years, climate literature has paid increasing attention to a new construct: climate strength, which refers to the degree of agreement among employees about climate within a work unit or organization. To further investigate the role climate plays in organizational outcomes, the current study examines the effects of climate strength on turnover at the organizational level. For that, we used a sample composed of 25,288 individuals from 150 medium and large-sized organizations from different industries and tested the direct and moderating effect climate strength has on five climate dimensions at the level of collective turnover. The results obtained showed that, after controlling for organizational climate quality, climate strength moderates the relationship between climate quality and collective turnover in three out of five climate facets. When climate strength is high, the influence of organizational climate on turnover is strengthened, but when climate strength is low, this influence weakens. However, there was no direct effect found between climate strength and collective turnover.
\end{abstract}

Keywords: organizational climate; climate strength; collective turnover; consensus of perceptions.

JEL Codes: D23, O15, J63. 


\section{Introdução}

O clima organizacional é formado pelas percepções partilhadas entre as pessoas sobre como elas experimentam seu ambiente de trabalho, a partir das práticas, políticas, procedimentos e rotinas organizacionais (Bowen \& Ostroff, 2004). Como tal, pode ser considerado um fenômeno de nível de grupo, já que é baseado nas percepções compartilhadas entre as pessoas. No entanto, como sua mensuração é feita no nível individual (clima psicológico) para então ser agregada no nível de grupo, isso significa que não apenas a qualidade dessas percepções (quão positivas elas são) importa, mas também o nível de consenso entre as percepções das pessoas desempenha papel importante na influência que o clima irá exercer em resultados organizacionais (Gonzáles-Romá \& Peiró, 2014; Lindell \& Brandt, 2000). Ao grau de uniformidade de percepções de um determinado grupo de pessoas sobre o clima, dáse o nome de força de clima (Chan, 1998; Gonzáles-Romá, Peiró, \& Tordera, 2002).

Esse construto é mais recente na literatura do assunto e, como tal, estudos que medem a influência de força de clima em alguma variável organizacional de interesse - seja no nível individual (ex: bemestar psicológico), grupal (ex: performance de time) ou organizacional (ex: desempenho financeiro) ainda apresentam conclusões mistas. Alguns estudos testaram o efeito direto de força de clima em resultados organizacionais (Bliese \& Halverson, 1998; Colquitt; Noe, \& Jackson, 2002; Gonzáles-Romá \& Peiró, 2014), mas os achados são pouco consistentes, com alguns poucos estudos que apresentam evidência de suporte, enquanto a maioria não. Um número relativamente maior de estudos de força de clima se concentra, ainda, em seu efeito moderador na relação de clima com algum resultado organizacional, mas também neste caso, apesar de haver maior evidência encontrada, nem todos eles apresentam efeitos significantes (Gonzáles-Romá \& Peiró, 2014; Schneider, Ehrhart, \& Macey, 2013; Zohar \& Luria, 2004).

Em paralelo a isso, dentre os resultados normalmente associados ao clima no nível organizacional, um número relativamente pequeno de estudos trabalha com turnover coletivo em si. Boa parte deles se concentra ainda em medição de atitudes coletivas (intenção de sair) e não necessariamente em nível de rotatividade de fato (Hom, Lee, Shaw, \& Hausknecht, 2017; Steel \& Lounsbury, 2009). E, quando turnover é efetivamente utilizado, os resultados são mistos, ora encontrando suporte para relação, ora não (Hancock, Allen, \& Soelberg, 2017). No caso específico de força de clima, os estudos são ainda mais escassos e sem suporte empírico encontrado (Sowinski, Fortmann, \& Lezotte, 2008). Ainda assim, turnover coletivo é um importante indicador de efetividade organizacional, seja porque reflete a capacidade de a empresa reter e acumular capital humano especializado (Shaw, Gupta, \& Delery, 2005), como também porque turnover coletivo já foi associado a inúmeros indicadores de desempenho organizacional (Hausknecht \& Trevor, 2011; Park \& Shaw, 2013).

Baseado nas lacunas mencionadas, neste trabalho pretende-se investigar o efeito de força de clima no turnover coletivo. Especificamente, usando diferentes dimensões de clima, pretende-se investigar o papel, direto e moderador, que a força de clima exerce em turnover em nível organizacional, para além da qualidade do clima dentro da organização.

Dentro deste escopo, o trabalho pretende fornecer algumas importantes contribuições: primeiro, ao contrário de estudos anteriores, que testaram essas relações usando normalmente escopos focados de clima (clima para justiça processual, clima para serviços, clima para segurança, clima ético, dentre outros), este trabalho explora não apenas dimensões diferentes de clima, como também testa seu poder preditivo sobre taxas de saída em nível organizacional, que é uma variável consequente distinta daquelas estudadas previamente. Segundo, boa parte dos estudos sobre força de clima tem foco em um nível de análise sub-organizacional, usando times ou unidades de trabalho/departamentos (Schneider et al., 2013). Poucos estudos exploram efeito de força de clima em nível organizacional, de tal forma que ainda não está claro se os efeitos encontrados em unidades organizacionais podem ser generalizados para o nível organizacional (González-Romá \& Piero, 2014). Especialmente porque, de acordo com a teoria da dispersão (Brown \& Kozlowski, 1999), construtos de nível organizacional ou suborganizacional, que são resultados da combinação de construtos individuais (como é o caso de clima organizacional) via 
processos de interação social, podem variar de forma significativa, de acordo com o tamanho da unidade organizacional em questão. O processo de emersão do clima dentro de um time de poucas pessoas é diferente do mesmo processo em uma organização com centenas ou milhares de pessoas. A interação entre as pessoas é menos intensa, menos uniforme e, portanto, a força de clima resultante apresentaria características diferentes. Assim, atendendo a uma lacuna levantada na literatura (González-Romá \& Piero, 2014), o presente trabalho investiga força de clima em nível organizacional, usando como unidade de análise a medição do ambiente de trabalho de empresas de médio e grande porte.

Terceiro, quando não baseiam a amostra em unidades organizacionais dentro de uma única empresa, os estudos usam amostras compostas por empresas de um único setor (normalmente de serviços, como bancos, varejo, hospitalar e hoteleiro). Diferentemente, este trabalho utilizou uma base diversa, robusta e heterogênea de 25.288 indivíduos advindos de 150 empresas de múltiplos setores e, como tal, é esperado que resultados sejam mais expressivos e de maior capacidade de generalização, que é uma demanda feita por estudos anteriores de clima (Schulte, Ostroff, Shmulyian, \& Kinicki, 2009). Além disto, ainda que haja estudos de clima no Brasil, todos eles trabalham com o conceito de qualidade de clima (Rocha \& Porto, 2012; Veloso, Dutra, \& Nakata, 2016), negligenciando o conceito de força de clima. Finalmente, ao testar o tipo de papel antecedente e moderador de força de clima em turnover coletivo, o trabalho avança na literatura de turnover, dado que a maioria dos trabalhos explora saída no nível individual. Como as teorias relacionadas ao turnover individual e a seus antecedentes mapeados não podem ser verticalmente replicados para abarcar adequadamente os processos coletivos envolvidos em taxas de saídas agregadas, estudos de metanálise recentes apontam que turnover no nível organizacional ainda permanece uma área de análise subdesenvolvida, com resultados inconsistentes, e que precisa de maior atenção e investigação (Hausknecht \& Trevor, 2011; Hom et al., 2017; Nyberg \& Ployhart, 2013; Park \& Shaw, 2013).

O trabalho se estrutura da seguinte forma: a próxima seção revisa a literatura usada para construir e apresentar as hipóteses. Na sequência, descrevem-se os procedimentos metodológicos usados na coleta, na mensuração e na análise dos dados e, em seguida, os resultados dos modelos de regressão são apresentados e discutidos. Por fim, as implicações do estudo, bem como suas limitações e pesquisas futuras, são apresentadas.

\section{Referencial Teórico}

\section{Força de clima: variância como construto focal}

A literatura de clima já demonstrou como o clima de determinada unidade organizacional se relaciona com importantes processos e resultados dessas unidades, como: performance financeira, inovação, comunicação, produtividade, dentre outros (Cropanzano, Li, \& Benson, 2011; GonzálesRomá, Fortes-Ferreira, \& Peiró, 2009; Schneider, Macey, Lee, \& Young, 2009; para revisão, ver Kuenzi \& Schminke, 2009).

Ao relacionar clima com resultados organizacionais, as pesquisas tipicamente agregam os escores individuais de clima (clima psicológico) em um construto de nível superior (clima organizacional). Nesse processo de agregação, modelos composicionais, que especificam a relação funcional entre construtos operacionalizados em níveis de análise diferentes, são empregados (Chan, 1998; James, 1982). Isso porque não se pode assumir que, ao compor variáveis de nível superior a partir de variáveis de nível inferior, os conceitos permanecerão isomórficos entre tais níveis. Com isso, em pesquisas multinível, esses modelos composicionais servem de guia não só para definir como construtos de diferentes níveis se relacionam conceitualmente, como também especificam como operacionalizar a transformação entre os níveis por meio de procedimentos de mensuração e análises estatísticas específicas (Chan, 2014). 
No caso de clima organizacional, usando a tipologia de modelos composicionais de Chan (1998), o modelo mais usado é o de consenso direto, em que o grau de consenso entre as percepções individuais dentro da organização é usado para justificar a agregação de dados individuais para representar um construto de nível superior, ou seja, certo consenso é condição necessária para a validade do construto em nível superior.

Neste modelo, o clima organizacional é operacionalizado pela média de percepções individuais sobre o ambiente de trabalho, mas o grau em que as percepções são compartilhadas entre os funcionários importa, ou seja, é preciso haver um grau mínimo de consenso nessas respostas, de forma que os escores individuais possam ser agrupados em um construto de nível superior. Inclusive, várias medidas de concordância e consistência entre avaliadores ( $r_{\mathrm{wg}}$ e ICCs) são usadas para verificar a validade dessas agregações (Bliese, 2000; Chan, 1998; James, Demaree, \& Wolf, 1984; Schneider et al., 2013).

No entanto, nos últimos anos, a pesquisa de clima passou a utilizar o grau de compartilhamento ou concordância de percepções entre funcionários de forma mais interessante, ao questionar qual o impacto da variabilidade de percepções dentro de unidades organizacionais nos resultados de clima. Essa linha de investigação abriu um novo tópico de pesquisa na literatura do assunto: força de clima, que se refere ao grau de concordância entre indivíduos a respeito de suas percepções sobre o clima da organização (Chan, 1998; Gonzáles-Romá et al., 2002; Schneider, Salvaggio, \& Subirats, 2002). Nessa abordagem, o grau de concordância dentro do grupo deixa de ser apenas um pré-requisito estatístico para comprovar que o construto de nível superior pode ser operacionalizado e a variabilidade dentro do grupo passa a ser tratada como um construto focal (Ostroff \& Fulmer, 2014). Portanto, diferentemente de clima, que é considerado um construto de consenso direto, força de clima é o que Chan denomina de um modelo composicional de dispersão, na medida em que a variância dentro do grupo deixa de ser tratada como variância do erro e passa a ser considerada como um atributo do grupo. Nesta linha, de acordo com a abordagem multinível proposta por Kozlowski e Klein (2000), enquanto clima organizacional, seria um construto resultante de um processo de emersão, cuja característica essencial é a convergência das medidas individuais no coletivo; força de clima seria um construto resultante de um processo de emersão diferente, cujo foco se baseia na descontinuidade e na variabilidade entre essas medidas no agregado.

Baseando-se nesses modelos composicionais e operacionalizada de forma consistente por meio de medidas de dispersão (variância, desvio padrão, desvio absoluto médio, coeficiente de variação), abriu-se caminho na literatura de clima para que uma nova linha de estudos passasse a se debruçar sobre a importância do consenso de percepções sobre o ambiente de trabalho, investigando tanto potenciais antecedentes de força de clima, como o papel que ela pode exercer em respostas afetivas e atitudinais, bem como em resultados organizacionais diversos (Colquitt et al., 2002; Gonzáles-Romá et al., 2002; Luria, 2008; Schneider et al., 2013; e para revisão, Gonzáles-Romá \& Peiró, 2014).

\section{A influência de força de clima em resultados organizacionais}

Por ser tópico mais recente na literatura de clima, há menor consenso sobre sua influência em determinados resultados organizacionais. A evidência empírica disponível aponta, ainda que de forma um pouco difusa, para um potencial papel moderador da força de clima para algumas dimensões de clima. Há também algum suporte, embora bem menor, sobre uma possível relação direta com algum resultado organizacional de interesse.

A respeito da relação direta de força de clima com resultados organizacionais, há poucos estudos que testam essa associação e os resultados empíricos são pouco consistentes, ora encontrando efeito em certos resultados atitudinais ou de performance, ora falhando em mostrar tais relações (Bliese \& Halverson, 1998; Colquitt et al., 2002; Gonzáles-Romá \& Peiró, 2014; Lindell \& Brandt, 2000; Sanders, Dorenbosch, \& Reuver, 2008). A justificativa teórica para sustentar essa relação é o paradigma da similaridade-atração (Byrne, 1971), que argumenta que as pessoas são atraídas por outras pessoas com quem elas percebem similaridade em determinados atributos, seja em termos de características demográficas, atitudes ou atributos de disposição. Tal similaridade se associaria à comunicação mais 
fluida, maior previsibilidade de comportamento e maior grau de concordância com o outro (Tsui \& O'Reilly, 1989), o que por sua vez produziria relações mais recompensadoras e menor distância social, facilitando a interação e o compartilhamento natural de visões sobre o ambiente social em que estão inseridas. Por essas razões, estudos prévios indicam que o grau de similaridade entre as pessoas, então, estaria positivamente associado à performance, embora eles tenham falhado em prover suporte empírico para essa relação direta (Colquitt et al., 2002).

No caso de força de clima e turnover coletivo, uma baixa similaridade ou menor convergência das visões compartilhadas entre as pessoas dentro da unidade organizacional revelaria, então, um grau menor de coesão entre os membros da organização. E, pelo modelo de atração-seleção-atrito (Schneider, 1987; Schneider, Goldstein, \& Smith, 1995), da mesma maneira que indivíduos se sentem atraídos por organizações cujos membros são similares, as pessoas tendem a sair das organizações com as quais elas percebem menor congruência. Portanto, um baixo consenso de percepções ou uma baixa força de clima geraria maior dissimilaridade entre as pessoas e maior percepção de incompatibilidade com a organização, gerando, por sua vez, maior rotatividade de pessoal.

Além disto, baseando-se na teoria da influência social (Festinger, 1950), pode-se ainda argumentar que o consenso entre as pessoas pode ser um indicador de qualidade do ambiente social do grupo em particular. Isso porque os grupos que apresentam maior consenso e coesão possuem membros com maior senso compartilhado de realidade social e, como consequência, apresentariam melhor funcionamento, maior identidade social e menor nível de conflito e estresse (Bliese \& Halverson, 1998). Nesta linha, em ambientes sociais mais positivos (de alto consenso), efeitos negativos de inevitáveis estressores no ambiente de trabalho seriam inclusive aliviados (Bliese \& Britt, 2001), o que contribuiria para menor rotatividade de pessoal na organização. Com isso, baseado nesses argumentos, tem-se a seguinte hipótese:

Hipótese 1: Há uma relação significante e negativa entre força de clima e turnover coletivo.

Boa parte dos estudos relacionados à força de clima trabalham seu papel moderador na relação entre clima e resultados organizacionais (Schneider et al., 2013). No entanto, os resultados também apresentam algumas discrepâncias. Alguns trabalhos não mostram um efeito moderador significante (Dawson, Gonzáles-Romá, Davis, \& West, 2008; Lindell \& Brandt, 2000; Potočnik, Tordera, MartínezTur, Peiró, \& Ramos, 2011; Rafferty \& Jimmieson, 2010; Sowinski et al., 2008; Zohar \& Luria, 2004), embora a maioria dos estudos ofereça suporte para tal efeito (Gonzáles-Romá \& Peiró, 2014, para revisão).

O modelo teórico que fundamenta a influência moderadora de força de clima no relacionamento entre o clima e determinado resultado organizacional é o conceito de força situacional de Mischel (1973), que se refere ao grau de ambiguidade presente em um determinado contexto. A ideia é que situações fortes são aquelas que levam os indivíduos a interpretar e a reagir a uma dada situação de forma similar, porque o contexto produz informação clara sobre o curso de ação mais apropriado. Portanto, em situações fortes, as reações são mais homogêneas (Meyer, Dalal, \& Hermida 2010). Em seu oposto, situações mais fracas levam à maior ambiguidade percebida a respeito de quais comportamentos são mais esperados ou apropriados e, nesses casos, traços individuais teriam uma influência maior no comportamento apresentado. Em situações fracas, as expectativas sobre o comportamento apropriado são menos consistentes em comparação com situações fortes.

Com isso, um clima forte geraria comportamentos mais similares, ao passo que um clima fraco levaria maiores diversidade e variabilidade de comportamentos. Por exemplo, usando esse argumento, Schneider, Salvaggio e Subirats (2002) testaram o efeito moderador de força de clima na relação entre clima para serviços e satisfação de clientes em agências bancárias. De acordo com os autores, quando a força de clima é alta, os funcionários apresentam comportamentos mais uniformes, gerando uma linha de frente coesa para os clientes. Com isso, os consumidores têm uma experiência de serviço mais consistente, independente da ocasião e dos funcionários com quem eles interagem. $\mathrm{E}$, de fato, os autores encontraram evidências, ainda que parciais para esse papel moderador, de forma que alta força de clima 
reforçou a influência do clima para serviços na satisfação dos consumidores, enquanto que, quando a força de clima era baixa, essa relação desapareceu.

Nesta mesma linha, outros estudos mostraram evidências de que clima fraco ou baixa força de clima, ao refletirem percepções idiossincráticas entre as pessoas e aumentarem a variabilidade de comportamentos apresentados, enfraquecem a relação entre clima e resultados organizacionais (Colquitt et al., 2002; González-Romá et al., 2002; Gonzáles-Romá et al., 2009; Van Vianen, Pater, Bechtoldt, \& Evers, 2011). Com isso, argumenta-se que força de clima como moderador ajuda, inclusive, a explicar o motivo pelo qual a magnitude do efeito da qualidade de clima em outra variável organizacional pode apresentar variações entre organizações (Chan, 2014).

Usando essa mesma lógica de força situacional no caso de turnover coletivo, embora estudos prévios já tenham fornecido evidências de uma associação significante entre avaliação global positiva dos funcionários sobre o ambiente de trabalho e a intenção de permanecer na empresa (Schulte et al., 2009) ou mesmo turnover em si (Hancock et al., 2017; Hom et al., 2017), qualquer benefício advindo de uma alta qualidade de clima na redução de turnover coletivo pode ser neutralizado ou atenuado por uma força de clima baixa. Especialmente porque, de acordo com alguns autores (Meyer et al., 2010; Schulte et al., 2009), essa maior variabilidade de percepções seria reflexo de um sistema pouco coerente ou menos coordenado de políticas e práticas organizacionais, que produziria um conjunto de mensagens ambíguas e inconsistentes para os funcionários sobre as prioridades da organização e dos comportamentos esperados, gerando dissonância cognitiva, um ambiente de trabalho menos favorável (Bowen \& Ostroff, 2004) e, como consequência, enfraquecendo a capacidade da qualidade de clima de reduzir níveis de turnover. Baseado nesses argumentos, tem-se a seguinte hipótese:

Hipótese 2: Força de clima apresenta um efeito moderador na relação entre clima organizacional e turnover coletivo, de forma que, quando a força de clima é alta, a influência do clima organizacional no turnover coletivo é reforçada, mas quando a força de clima é baixa, essa influência se enfraquece.

\section{Método}

\section{Amostra e procedimentos}

Os dados usados no estudo foram coletados como parte de uma pesquisa realizada em 2014 por um Instituto de Pesquisa, cujo objetivo foi verificar as práticas de gestão e o clima organizacional para publicação de uma pesquisa anual sobre qualidade do ambiente de trabalho das empresas.

Os dados foram coletados a partir de dois instrumentos: um questionário sobre a qualidade do ambiente de trabalho da empresa, formado por 67 afirmativas usando uma escala Likert de 5 pontos (de 1 - discordo totalmente a 5 - concordo totalmente), que visa mapear como o funcionário percebe o ambiente de trabalho; e um outro questionário sobre a qualidade da gestão de pessoas da empresa, que busca mapear as práticas e as políticas de gestão de pessoas da empresa pesquisada. O primeiro instrumento é respondido voluntariamente pelos funcionários da empresa pesquisada por meio de questionário anônimo, e o segundo instrumento é obtido por formulários eletrônico respondidos pelo responsável pela área de RH da empresa pesquisada. Os dados usados nesta pesquisa foram aqueles coletados pelo questionário respondido pelos funcionários.

A amostra do presente estudo contou com 25.288 respondentes válidos de 150 organizações que participaram dessa pesquisa anual. Do total de empresas, $48 \%$ eram do setor industrial, $43 \%$ de serviços e $9 \%$ de comércio.

Segundo critérios ad-hoc definidos pelo Instituto de Pesquisa responsável pela coleta, para poder participar da pesquisa, a empresa precisava ter, ao menos, 100 funcionários. Além disto, visando obter 
representatividade em cada organização pesquisada, a empresa só era considerada na pesquisa se o número de respondentes fosse satisfatório em relação ao cálculo de tamanho mínimo de amostra, com $95 \%$ de confiança e $5 \%$ de margem de erro.

\section{Mensuração das variáveis e agregação}

Dimensões de clima organizacional: uma análise fatorial exploratória foi feita utilizando a matriz de correlações dos 67 itens do questionário de clima, via método das componentes principais, e foram extraídos oito fatores utilizando o critério de ter pelo menos $70 \%$ da variabilidade total dos itens explicada pelos fatores (70,2\% de variabilidade explicada). Além disso, foram calculados os escores fatoriais rotacionados via VARIMAX para facilitar a interpretação dos resultados. Dos oito fatores, três deles foram descartados da análise, porque os índices de concordância (ICCs) ficaram aquém de parâmetros aceitáveis para agregação. Assim, permaneceram na análise as seguintes cinco dimensões de clima: (a) Suporte gerencial, que se relaciona à qualidade percebida das competências do(a) gestor(a) perante o indivíduo e sua equipe; (b) Identificação com empresa, compreendida por orgulho, senso de pertencimento, concordância com os objetivos e estratégia da empresa, bem como seu impacto na sociedade; (c) Relacionamento, ou seja, como são o ambiente social e a relação do indivíduo com outras pessoas, da sua área ou não; (d) Recompensa, cujas percepções estão relacionadas com a troca do seu trabalho por remuneração e recompensas não financeiras; (e) Crescimento, que está ligado à carreira e às perspectivas de desenvolvimento profissional na organização. A Tabela 1 apresenta os exemplares de itens, os números de itens e o alfa de cada dimensão de clima.

Tabela 1

\section{Dimensões de Clima e Exemplares de Itens}

\begin{tabular}{lccl}
\hline Dimensão & $\mathbf{N}^{\mathbf{0}}$ itens & Alfa & Exemplos de itens \\
\hline Suporte Gerencial & 20 & 0.972 & $\begin{array}{l}\text { Sempre que preciso, posso contar com meu chefe para assuntos } \\
\text { pessoais e profissionais. } \\
\text { Meu chefe conhece profundamente sua área de atuação. }\end{array}$ \\
\hline $\begin{array}{l}\text { Identificação com } \\
\text { empresa }\end{array}$ & 16 & 0.952 & $\begin{array}{l}\text { Essa organização tem um imenso significado pessoal por mim. } \\
\text { Conheço os objetivos da empresa em que trabalho e concordo com } \\
\text { eles. }\end{array}$ \\
\hline Relacionamento & 8 & 0.921 & $\begin{array}{l}\text { Meus colegas estão sempre dispostos a dividir comigo o que sabem. } \\
\text { Nesta empresa, as pessoas estão sempre dispostas a ajudar umas às } \\
\text { outras. }\end{array}$ \\
\hline Recompensa & 5 & 0.909 & $\begin{array}{l}\text { Considero justo o salário pago por esta empresa aos seus } \\
\text { funcionários. } \\
\text { Sou recompensado, de maneira justa, pela qualidade do trabalho que } \\
\text { apresento. }\end{array}$ \\
\hline Crescimento & 8 & 0.916 & $\begin{array}{l}\text { Acredito que, trabalhando nesta empresa, terei oportunidade de fazer } \\
\text { carreira e crescer. } \\
\text { Sei o que devo fazer para crescer profissionalmente nesta empresa. }\end{array}$ \\
\hline
\end{tabular}

Força de clima: a força de clima para cada dimensão de clima resultante da análise fatorial foi calculada pela medida $\mathrm{AD}_{\mathrm{M}}$ de desvio absoluto médio usada por Burke, Finkelstein e Dusig (1999), segundo a equação a seguir:

$$
A D_{M}=\frac{\sum_{i=1}^{N}\left|x_{i}-\bar{x}\right|}{N}
$$

em que $x_{i}$ representa o escore de clima individual, e $\overline{\bar{x}}$ representa a média global dos escores de clima. O valor resultante foi então multiplicado por -1 , como feito anteriormente em outros estudos 
(Dawson et al., 2008; Gonzáles-Romá et al., 2002). Com isso, tem-se concordância entre sinal e sentido, de modo que, quanto maior o escore em $\mathrm{AD}_{\mathrm{M}}$, maior é a concordância nas percepções sobre aquela dimensão de clima da empresa, ou seja, mais forte é o clima.

Turnover: índice de rotatividade de funcionários, foi calculado pela divisão do total de saídas no ano sobre a quantidade total de empregados no mesmo ano.

Controles: foram adicionadas três variáveis controle aos modelos porte da empresa, que foi medido pelo número de funcionários; setor, onde as empresas da amostra foram divididas nos setores de serviços, indústria e comércio e origem de capital, seja ele nacional ou estrangeiro.

Para que fosse possível agregar os escores individuais (clima psicológico) em um nível de análise organizacional (clima organizacional), medidas de confiabilidade e concordância entre avaliadores largamente utilizadas na literatura de clima foram calculadas para garantir que a agregação pudesse ser feita (James, 1982). Na Tabela 2, tem-se os valores das correlações intraclasse ICC(1) e ICC(2) para verificar confiabilidade na diferença entre grupos, assim como os valores de concordância $r_{w g}$ em cada uma das escalas. Os resultados mostram-se satisfatórios, com bons níveis de confiabilidade, com valores de ICC(2) bem acima do parâmetro de 0.7 e valores de ICC(1) de 0.06 a 0.17 , que estão dentro de intervalos aceitáveis para este índice (Bliese, 2000; LeBreton \& Senter, 2008). Já para a medida de concordância, os valores médios de $r_{\text {wg }}$ estão todos acima da nota de corte usual de 0.7 , à exceção de recompensa. Porém, como as outras medidas mostraram bons ajustes, essa dimensão foi mantida na análise.

Tabela 2

Correlações Intraclasse, Estatísticas $\mathrm{AD}_{\mathrm{M}}$ e $\mathbf{r}_{\mathrm{wg}}$ para as Dimensões de Clima

\begin{tabular}{lcccc}
\hline Dimensão & ICC(1) & ICC $(2)$ & Média $\mathrm{r}_{\mathrm{wg}}$ (intervalo) & Média $\mathrm{AD}_{\mathrm{M}}$ (intervalo) \\
\hline Suporte Gerencial & 0.17 & 0.97 & $0.92(0.43-0.97)$ & $-0.66(-1.02-00)$ \\
Identificação com empresa & 0,11 & 0.95 & $0.95(0.70-0.99)$ & $-0.51(-0.84-00)$ \\
Relacionamento & 0.07 & 0.92 & $0.89(0.66-0.99)$ & $-0.59(-0.84-00)$ \\
Recompensa & 0.06 & 0.91 & $0.61(0.19-0.94)$ & $-0.82(-1.09-00)$ \\
Crescimento & 0.06 & 0.92 & $0.88(0.32-0.99)$ & $-0.60(-0.95-00)$ \\
\hline
\end{tabular}

Nota. $\mathrm{K}=168$ para $\operatorname{ICC}(1)$, equivale ao $\mathrm{n}^{\circ}$ médio de respondentes por empresa.

\section{Resultados}

As médias, desvios padrões e correlações entre as medidas de clima, força de clima e turnover são apresentadas na Tabela 3. Pode-se observar que todas as dimensões de clima apresentaram relação negativa com turnover, como esperado. Embora as correlações entre as medidas de clima sejam relativamente altas, a análise fatorial exploratória e as medidas de confiabilidade indicam a existência de diferentes construtos dentro da escala completa de clima. 


\section{Tabela 3}

Médias, Desvios Padrão e Correlações entre as Variáveis do Estudo (n=150)

\begin{tabular}{|c|c|c|c|c|c|c|c|c|c|c|c|c|c|}
\hline Variáveis & Média & DP & 1 & 2 & 3 & 4 & 5 & 6 & 7 & 8 & 9 & 10 & 11 \\
\hline 1. Suporte Gerencial & 4.04 & 0.27 & 1 & & & & & & & & & & \\
\hline 2. Identif. Empresa & 4.31 & 0.28 & $0.869^{* *}$ & 1 & & & & & & & & & \\
\hline 3. Relacionamento & 4.00 & 0.31 & $0.897^{* *}$ & $0.862^{* *}$ & 1 & & & & & & & & \\
\hline 4. Recompensa & 3.65 & 0.41 & $0.874^{* *}$ & $0.903^{* *}$ & $0.861^{* *}$ & 1 & & & & & & & \\
\hline 5. Crescimento & 4.16 & 0.28 & $0.934^{* *}$ & $0.948^{* *}$ & $0.882^{* *}$ & $0.912^{* *}$ & 1 & & & & & & \\
\hline 6. FC Suporte Gerencial & -0.66 & 0.13 & $0.545^{* *}$ & $0.340^{* *}$ & $0.300^{* *}$ & $0.297^{* *}$ & $0.436^{* *}$ & 1 & & & & & \\
\hline 7. FC Identif. Empresa & -0.51 & 0.12 & $0.537^{* *}$ & $0.562^{* *}$ & $0.379^{* *}$ & $0.425^{* *}$ & $0.566^{* *}$ & $0.822^{* *}$ & 1 & & & & \\
\hline 8. FC Relacionamento & -0.59 & 0.10 & $0.377^{* *}$ & $0.254^{* *}$ & $0.274^{* *}$ & $0.170^{*}$ & $0.317^{* *}$ & $0.841^{* *}$ & $0.820^{* *}$ & 1 & & & \\
\hline 9. FC Recompensa & -0.82 & 0.14 & $0.342^{* *}$ & $0.274^{* *}$ & $0.170^{*}$ & $0.293^{* *}$ & $0.332^{* *}$ & $0.796^{* *}$ & $0.826^{* *}$ & $0.822^{* *}$ & 1 & & \\
\hline 10. FC Crescimento & -0.60 & 0.13 & $0.535^{* *}$ & $0.452^{* *}$ & $0.329^{* *}$ & $0.383^{* *}$ & $0.558^{* *}$ & $0.872^{* *}$ & $0.917^{* *}$ & $0.817^{* *}$ & $0.827^{* *}$ & 1 & \\
\hline 11. Turnover & 0.18 & 0.14 & $-0.087^{*}$ & $-0.101^{*}$ & $-0.089^{*}$ & $-0.127^{*}$ & $-0.117^{*}$ & 0.036 & 0.011 & $0.087^{*}$ & 0.072 & -0.009 & 1 \\
\hline
\end{tabular}

Nota. $\mathrm{FC}=$ força de clima

$* \mathrm{p}<0.05 \mathrm{e}^{* *} \mathrm{p}<0.01$ 
Aplicou-se então um modelo de regressão linear generalizado utilizando a distribuição gaussiana inversa. A escolha de tal distribuição foi feita com base no comportamento do turnover, com forte assimetria positiva, ou seja, concentração nos menores valores. Assim, o uso desse modelo objetivou apresentar um ajuste relativamente melhor comparado ao modelo de regressão com erros normais (mesmo usando o log do turnover em um modelo de regressão usual, os erros não apresentaram normalidade).

Além disto, como estudos prévios (Bliese \& Halverson, 1998; Gonzáles-Romá \& Peiró, 2014) alertam, uma vez que a força de clima se baseia em medida de dispersão (no caso, desvio absoluto médio) e o clima organizacional é representado pela média de percepções de clima, ambos são interdependentes. De fato, para todas as dimensões de clima, as duas medidas são positivamente correlacionadas, conforme Tabela 3. Por esse motivo, cada preditor foi inserido hierarquicamente na regressão, de forma a avaliar se a adição da variável produziria um aumento significativo na qualidade do modelo ( seudo $\mathrm{R}^{2}$ ). Também foi testada a contribuição de cada variável no modelo e, para tanto, foi utilizado um teste que se baseia nas funções desvios (deviance) para produzir estatísticas de teste equivalentes a um F-parcial, isso porque os modelos lineares generalizados não possuem $\mathrm{R}^{2}$, pois são estimados por máxima verossimilhança (Casella \& Berger, 2011).

A Tabela 4 apresenta os resultados da regressão para cada dimensão de clima. A validade das suposições do modelo de regressão foram avaliadas com base numa cuidadosa análise de resíduos. Os erros apresentaram heterocedasticidade, então, houve necessidade de usar erros padrões robustos. Não foram observados valores muito extremos, de forma que todas as empresas foram consideradas. No caso da qualidade do ajuste dos modelos lineares generalizados, a avaliação foi feita calculando-se o pseudo $\mathrm{R}^{2}$ pelo método de McFadden.

Tabela 4

Modelo Linear Generalizado de Turnover com Clima e Força de Clima ( $\mathbf{N}=150)$

\begin{tabular}{|c|c|c|c|c|c|c|}
\hline & Escala de clima & $\begin{array}{l}\text { Suporte } \\
\text { Gerencial }\end{array}$ & $\begin{array}{l}\text { Identificação } \\
\text { com Empresa }\end{array}$ & Relacionamento & Recompensa & Crescimento \\
\hline \multirow[t]{4}{*}{ (1) } & Clima sozinho & & & & & \\
\hline & $\operatorname{Clima}(\beta)$ & $-0,06^{* *}$ & $-0,08^{*}$ & $-0,07 * *$ & $-0,05 * *$ & $-0,07 *$ \\
\hline & $\mathrm{R}^{2}$ Ajustado (McFadden) & 0,06 & 0,07 & 0,06 & 0,02 & 0,03 \\
\hline & Teste F (deviance) & $4,26 * *$ & $3,59^{*}$ & $4,13 * *$ & $2,83^{*}$ & $3,54^{*}$ \\
\hline \multirow[t]{5}{*}{ (2) } & Força de clima adicionada & & & & & \\
\hline & Clima $(\beta)$ & $-0,09 *$ & $-0,13^{*}$ & $-0,08 *$ & $-0,07 * *$ & $-0,10^{*}$ \\
\hline & Força de clima ( $\beta$ ) & 0,11 & 0,13 & 0,06 & 0,12 & 0,06 \\
\hline & $\mathrm{R}^{2}$ Ajustado (McFadden) & 0,05 & 0,07 & 0,05 & 0,01 & 0,03 \\
\hline & Teste F (deviance) & 0,23 & 0,68 & 0,19 & 0,16 & 0,18 \\
\hline \multirow[t]{6}{*}{ (3) } & Termo de Interação adicion & & & & & \\
\hline & Clima $(\beta)$ & $-0,28 * *$ & $-0,29 *$ & $-0,26^{* *}$ & $-0,26^{* *}$ & $-0,21 *$ \\
\hline & Força de clima $(\beta)$ & $1,15^{* *}$ & 1,27 & $1,22^{*}$ & $0,97^{*}$ & 0,78 \\
\hline & Interação ( $\beta)$ & $-0,24 * * *$ & $-0,25$ & $-0,27 * *$ & $-0,21 * *$ & $-0,16$ \\
\hline & $\mathrm{R}^{2}$ Ajustado (McFadden) & 0,09 & 0,06 & 0,08 & 0,05 & 0,04 \\
\hline & Teste F (deviance) & $6,85^{* * *}$ & 0,51 & $5,26 * *$ & $4,04 * *$ & 1,42 \\
\hline
\end{tabular}

Nota. Controles: porte, setor e origem de capital.

$* \mathrm{p}<0,10 ; * * \mathrm{p}<0,05 \mathrm{e} * * * \mathrm{p}<0,01$. 
Primeiro, por um conjunto de regressões, testou-se o efeito direto de cada dimensão de clima no turnover. Na etapa 2, adicionou-se a variável força de clima para verificar se ela tinha um efeito aditivo em turnover, para além do efeito da média de clima. Em uma terceira etapa, adicionou-se o termo de interação entre clima e força de clima. A Tabela 4 apresenta os resultados das regressões.

Os resultados mostram relação negativa significante entre cada dimensão de clima e turnover, sinalizando que a percepção positiva dos funcionários sobre essas facetas do ambiente de trabalho contribui para diminuir a rotatividade nas empresas. No entanto, ao se inserir força de clima na etapa 2 do modelo, ela não apresentou efeito no turnover em nenhuma das dimensões de clima. Com isto, não há suporte para a Hipótese 1.

As Figuras 1 e 2 mostram o efeito moderador de força de clima na relação entre clima e turnover para Suporte Gerencial e Relacionamento, respectivamente. Em ambos os gráficos, é possível observar que, quanto maior o escore da força de clima, maior é a inclinação da reta. Assim, tem-se que, quando a força de clima é alta, o efeito do clima no turnover é reforçado, ao passo que a força de clima baixa enfraquece essa relação. Esses resultados fornecem suporte para a Hipótese 2.

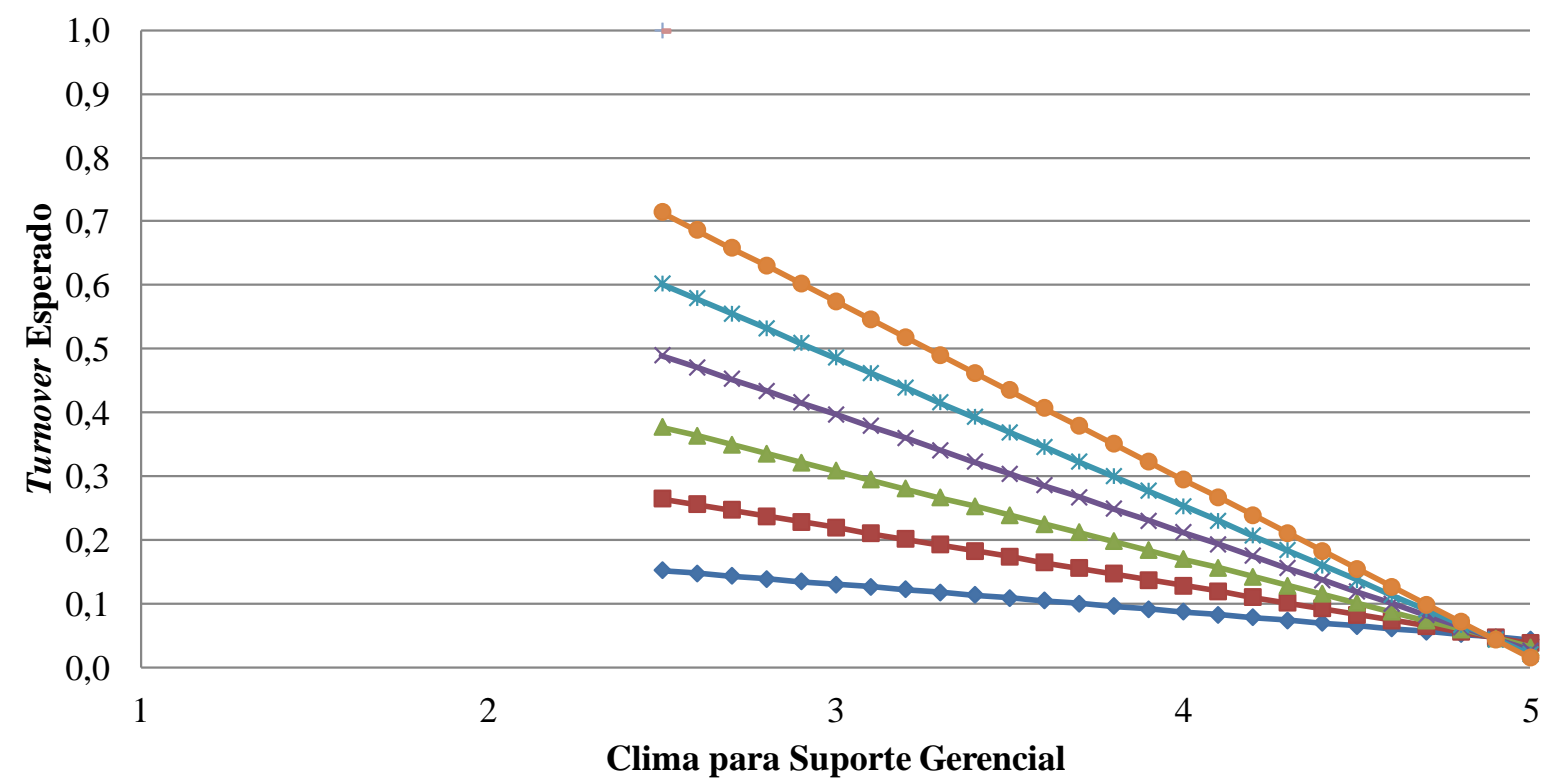

$$
\begin{aligned}
& \rightarrow \text { AD_Lid }=-1 \rightarrow \text { AD_Lid }=-0,8 \leftarrow \text { AD_Lid }=-0,6 \\
& * \text { AD_Lid }=-0,4 \rightarrow \text { AD_Lid }=-0,2-\text { AD_Lid }=0
\end{aligned}
$$

Figura 1. Efeito Moderador da Força de Clima para Suporte Gerencial na Relação Clima para Suporte Gerencial e Turnover 


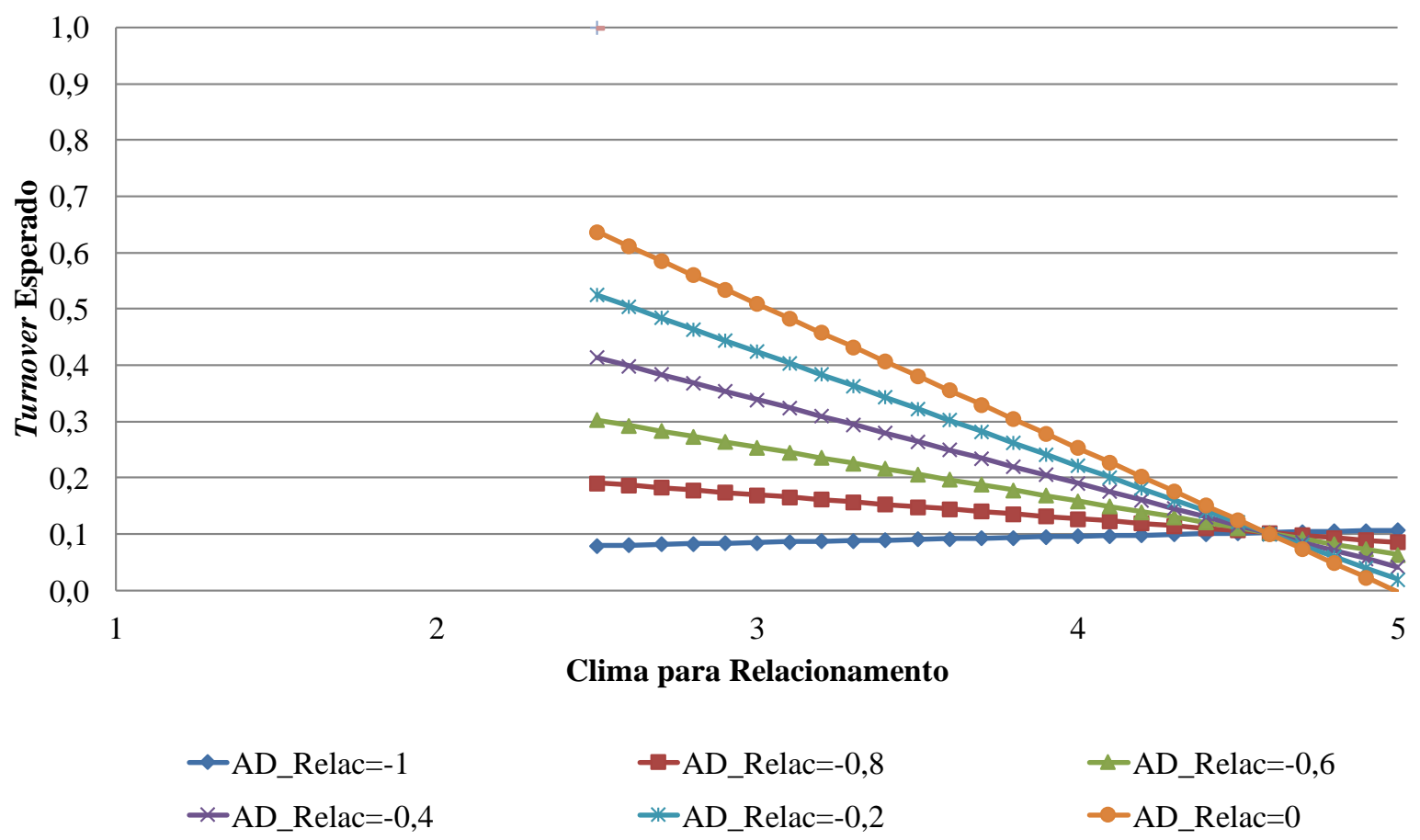

Figura 2. Efeito moderador da força de clima para Relacionamento na relação clima para Relacionamento e turnover

Sobre o termo de interação entre clima e força de clima, não se observa efeito para duas das cinco dimensões de clima analisadas: Identificação com a Empresa e o Crescimento. No entanto, para Suporte Gerencial, Relacionamento e Recompensa, a interação é significante, sinalizando que a força de clima modera a relação entre clima e turnover para essas dimensões. O termo de interação gera de $2 \%$ (para Relacionamento) a 3\% (para Suporte Gerencial e Relacionamento) de variância adicional explicada sobre a média da dimensão de clima sobre turnover.

\section{Discussão}

O trabalho buscou investigar o papel que força de clima exerce no turnover em nível organizacional, a partir de uma base heterogênea de 150 empresas de médio e grande porte de múltiplos setores no Brasil. Os resultados obtidos oferecem algumas contribuições importantes para o entendimento do efeito de força de clima em um resultado de nível organizacional, considerando que há uma lacuna existente na literatura de força de clima neste nível de análise (González-Romá \& Piero, 2014; Schneider et al., 2013). Além disto, o trabalho também avança no entendimento da interação existente entre qualidade de clima e consenso sobre o clima nas taxas de rotatividade de funcionários da organização, que é um indicador importante para avaliar a efetividade de práticas de gestão de pessoas da organização, bem como revela sua capacidade de reter capital humano e social (Dess \& Shaw, 2001; Shaw et al., 2005).

Os resultados (Tabela 4) apontaram que força de clima não apresenta efeito direto nas taxas de rotatividade. Tal achado se alinha de forma geral com estudos prévios, que também não encontraram relação direta entre força de clima e resultados organizacionais mais tangíveis, como performance e absenteísmo (Colquitt et al., 2002).

Assim, o fato de força de clima em si não gerar efeito direto no turnover pode ser explicado pelo fato de que, ainda que maior nível de concordância de percepções, possa fornecer maior noção de similaridade e compatibilidade entre as pessoas e se relacionar ao maior bem-estar psicológico (Bliese 
\& Halverson, 1998), alto compartilhamento de percepções (força de clima alta) sozinho não implica necessariamente que as pessoas estejam enxergando um ambiente de trabalho saudável que as encoraje a permanecer na empresa. De fato, alguns autores apontam que qualquer influência da similaridade das percepções dos indivíduos em suas respostas em relação à organização dependeria do tom avaliativo do consenso dessas percepções (Van Vianen et al., 2011). O argumento é que, se as percepções compartilhadas sobre o ambiente de trabalho são negativas, mesmo que haja alto consenso entre elas (alta força de clima), as reações seriam negativas. Por exemplo, pode ocorrer que haja uma alta força de clima para a dimensão de Relacionamento, mas se o escore médio dessa faceta for baixo, então há apenas consenso que o relacionamento interpessoal entre os membros da organização é pobre e, com isso, força de clima nessa dimensão, pouco ajudaria a reduzir as taxas de turnover. Deste modo, um alto escore de clima na dimensão é uma necessidade também para gerar efeito nas taxas de rotatividade. Nesta linha de argumentação, o efeito que a força de clima pode exercer nos resultados organizacionais depende da direção do consenso entre as pessoas.

Se a força de clima sozinha não apresenta efeito direto no turnover, a qualidade do clima por si já importa. De forma geral, acredita-se que percepções gerais mais positivas de clima levam a taxas de rotatividade menores nas empresas. $\mathrm{E}$, de fato, os resultados deste trabalho corroboram este argumento. Os escores médios de clima em todas as dimensões de clima estudadas apresentaram efeito significante em turnover.

Porém, um ponto fundamental a se observar no efeito do clima organizacional em turnover é que a magnitude desse efeito depende da força de clima, ou seja, a influência da qualidade do clima organizacional na redução nos níveis de turnover coletivo depende da coesão e do consenso das percepções entre os indivíduos sobre o ambiente de trabalho. No trabalho, das cinco dimensões de clima analisadas, a força de clima de três delas (Suporte Gerencial, Recompensas e Relacionamento) apresentou efeito moderador significante entre seus respectivos escores de clima e turnover, de acordo com a Tabela 4.

Portanto, esses resultados sugerem que não é apenas a percepção agregada positiva do ambiente de trabalho que importa, mas, especialmente, o consenso das percepções entre os membros da organização. Assim, percepções positivas de clima sobre o ambiente de trabalho, quando não acompanhadas de alto consenso entre seus membros, não necessariamente levam à menor rotatividade da força de trabalho. Como os resultados evidenciaram, o grau de concordância entre os funcionários a respeito das suas percepções sobre o clima da organização tem um efeito moderador importante entre qualidade de clima e turnover.

Em resumo, embora haja muita preocupação das organizações no sentido de assegurar um clima positivo para auferir seus vários benefícios em uma miríade de resultados organizacionais, atenção igual precisa ser direcionada para fomentar a força do clima na empresa, dado que a intensidade do efeito do clima na redução do turnover coletivo depende do nível de uniformidade das percepções entre os indivíduos sobre o ambiente de trabalho. Nesse sentido, de um ponto de vista prático, os resultados deste trabalho apontam que, qualquer iniciativa que intencione reduzir as taxas de saída de funcionários, precisa concentrar esforços para promover não apenas políticas e rotinas organizacionais que melhorem a qualidade do clima na organização, mas também assegurar maneiras para que as mensagens emitidas por meio dessas práticas organizacionais sejam comunicadas de forma clara e por vários canais, de forma a gerar maior homogeneidade de percepção entre os membros da organização.

\section{Limitações e Pesquisas Futuras}

Apesar das contribuições deste trabalho, algumas considerações sobre suas limitações precisam ser feitas e podem, inclusive, balizar futuros estudos. Primeiro, ao usar os dados disponíveis de uma pesquisa anual de percepções sobre qualidade do ambiente de trabalho, as dimensões de clima foram derivadas empiricamente. Se, por um lado, isso permitiu trabalhar com um vasto número de empresas 
de múltiplos setores, gerando maior generalização dos resultados em relação aos estudos anteriores feitos em setores específicos (Dawson et al., 2008; Potočnik et al., 2011; Schneider et al., 2002); por outro lado, ela levou ao uso de dimensões genéricas de clima, que é uma abordagem criticada pela sua falta de foco e parcimônia, o que prejudicaria sua capacidade de capturar e predizer o efeito de clima na variável resposta de interesse (Schneider, 1975; Schneider et al., 2013). O argumento é que, de acordo com os resultados a medir, os estudos de clima deveriam adotar uma abordagem estratégica, em que a medida de clima é específica e focada no resultado, como clima para serviços, segurança, ética, dentre outros (Ostroff, Kinicki, \& Muhammad, 2013; Schneider, Gonzáles-Romá, Ostroff, \& West, 2017). Neste estudo, como turnover coletivo é um resultado organizacional de amplo espectro, o não uso de facetas estratégicas de clima é menos problemático, mas ainda não deixa de ser uma limitação.

Segundo, neste estudo, o clima e as taxas de turnover foram medidos no mesmo ano. No entanto, pode ser que haja um efeito defasado na relação entre força de clima e turnover coletivo. Por exemplo, medindo o efeito de força de clima para serviços e qualidade de serviço, estudos similares encontraram resultados diferentes na relação, ao medir qualidade do serviço 3 meses depois (Schneider et al., 2002) ou 3 anos depois (Potočnik et al., 2011) da medição de força de clima. Assim, um estudo longitudinal em que tanto o clima como as taxas de turnover fossem medidos mais vezes no tempo poderia capturar eventuais efeitos defasados na relação. E, com isto, não apenas contribuir de forma significativa para o entendimento sobre como a força de clima evolui ao longo do tempo, mas também sobre seus impactos de médio prazo, dada a forte escassez de estudos longitudinais relacionados à força de clima (GonzálezRomá \& Piero, 2014).

Por fim, parte da argumentação derivada do paradigma da similaridade-atração (Byrne, 1971) para as possíveis relações entre força de clima e turnover envolve a capacidade de o nível de consenso entre as percepções dos indivíduos influenciar comunicação e conflito entre as pessoas. Porém, esses processos não puderam ser examinados adequadamente neste estudo.

Estudos futuros poderiam ainda se focar na inclusão de possíveis mediadores atitudinais na relação entre força de clima e turnover coletivo, de forma a realizar uma análise de mediação que permita clarificar os mecanismos causais a partir dos quais o consenso de percepções sobre o ambiente de trabalho afeta a rotatividade organizacional (Hayes, 2017; Iacobucci, 2008). Na literatura de turnover, dentre os possíveis antecedentes, aponta-se que atitudes coletivas (como satisfação no trabalho e comprometimento organizacional) são importantes preditores de turnover (Hausknecht \& Trevor, 2011; Steel \& Lounsbury, 2009). Além disto, estudos prévios de força de clima já mostraram suporte para seu efeito em resultados afetivos e atitudinais (Gonzáles-Romá \& Peiró, 2014). Dados esses resultados prévios, pode ser válido examinar então o efeito indireto que força de clima exerce no turnover por meio de certas atitudes coletivas.

\section{Material Suplementar}

Todos os dados e materiais foram disponibilizados publicamente por meio da plataforma Mendeley e podem ser acessados em: Nakata, Lina Eiko; Iwai, Tatiana; Bruscato Bortoluzzo, Adriana (2018), "Data for: "' published by RAC-Revista de Administração Contemporânea", Mendeley Data, v1http://dx.doi.org/10.17632/f3m2r2g994.1

\section{Contribuições}

$1^{\circ}$ autor: definição do escopo da pesquisa, revisão da literatura, análise de dados, discussão dos resultados, redação do manuscrito, revisão de todas as fases do manuscrito.

$2^{\circ}$ autor: definição do escopo da pesquisa, modelagem e análise de dados, discussão dos resultados.

$3^{\circ}$ autor: coleta e organização dos dados, revisão de todas as fases do manuscrito.

$4^{\circ}$ autor: coleta e organização dos dados. 


\section{Referências}

Bliese, P. D. (2000). Within-group agreement, non-independence, and reliability: Implications for data aggregation and analyses. In K. J. Klein \& S. W. J. Kozlowski (Eds.), Multilevel theory, research and methods in organizations: Foundations, extensions, and new directions (pp. 349-381). San Francisco: Jossey-Bass.

Bliese, P. D., \& Britt, T. W. (2001). Social support, group consensus and stressor-strain relationships: Social context matters. Journal of Organizational Behavior, 22(4), 425-436. http://doi.org/10.1002/job.95

Bliese, P. D., \& Halverson, R. R. (1998). Group consensus and psychological well-being: A large field study. Journal of Applied Psychology, 28(7), 563-580. http://doi.org/10.1111/j.15591816.1998.tb01720.x

Bowen, D., \& Ostroff, C. (2004). Understanding HRM - firm performance linkages: The role of the strength of the HRM system. Academy of Management Review, 29(2), 203-211. http://doi.org/10.5465/AMR.2004.12736076

Brown, K. G., \& Kozlowski, S. W. J. (1999). Dispersion theory: Moving beyond a dichotomous conceptualization of emergent organizational phenomena. Paper presented at the Annual Meeting of the Society of Industrial and Organizational Psychology, Atlanta, GA, USA, 14.

Burke, M. J., Finkelstein, L. M., \& Dusig, M. S. (1999). On average deviation indices for estimating interrater agreement. Organizational Research Methods, 2(1), 49-68. https://doi.org/10.1177/109442819921004

Byrne, D. (1971). The attraction paradigm. New York: Academic Press.

Casella, G., \& Berger, R. L. (2001). Statistical inference (2nd ed.). Pacific Grove: Duxbury Press.

Chan, D. (1998). Functional relations among constructs in the same contend domain at different levels of analysis: A typology of composition models. Journal of Applied Psychology, 83(2), 234-246. http://doi.org/10.1037/0021-9010.83.2.234

Chan, D. (2014). Multilevel and aggregation issues in climate and culture research. In B. Schneider \& K. M. Barbera (Eds.), The Oxford Handbook of Organizational Climate and Culture (pp. 484495). New York, NY: Oxford University Press.

Colquitt, J. A., Noe, R. A., \& Jackson, C. L. (2002). Justice in teams: Antecedents and consequences of procedural justice climate. Personnel Psychology, 55(1), 83-109. http://doi.org/10.1111/j.17446570.2002.tb00104.x

Cropanzano, R., Li, A., \& Benson, L., III (2011). Peer justice and teamwork process. Group \& Organization Management, 36(5), 567-596. https://doi.org/10.1177/1059601111414561

Dawson, J. F., Gonzáles-Romá, V., Davis, A., \& West, M. A. (2008). Organizational climate and climate strength in UK hospitals. European Journal of Work and Psychology, 17(1), 89-111. http://doi.org/10.1080/13594320601046664

Dess, G. G., \& Shaw, J. D. (2001). Voluntary turnover, social, capital, and organizational performance. Academy of Management Review, 26(3), 446-456. http://doi.org/10.5465/AMR.2001.4845830

Festinger, L. (1950). Informational social comparison. Psychological Review, 57, 271-282. 
Gonzáles-Romá, V., Fortes-Ferreira, L., \& Peiró, J. M. (2009). Team climate, climate strength and team performance. A longitudinal study. Journal of Occupational and Organizational Psychology, 82(3), 511-536. http://doi.org/10.1348/096317908X370025

Gonzáles-Romá, V., \& Peiró, J. M. (2014). Climate and culture strength. In B. Schneider, K. M. Barbera (Eds.), The Oxford handbook of organizational climate and culture (pp. 496-531). New York, NY: Oxford University Press.

Gonzáles-Romá, V., Peiró, J. M., \& Tordera, N. (2002). An examination of the antecedents and moderator influences of climate strength. Journal of Applied Psychology, 87(3), 465-473. http://doi.org/10.1037/0021-9010.87.3.465

Hancock, J. I., Allen, D. G., \& Soelberg, C. (2017). Collective turnover: An expanded meta-analytic exploration and comparison. Human Resource Management Review, 27(1), 61-86. https://doi.org/10.1016/j.hrmr.2016.06.003

Hausknecht, J. P., \& Trevor, C. O. (2011). Collective turnover at the group, unit, and organizational levels: Evidence, issues, and implications. Journal of Management, 37(1), 352-388. https://doi.org/10.1177/0149206310383910

Hom, P. W., Lee, T. W., Shaw, J. D, \& Hausknecht, J. P. (2017). One hundred years of employee turnover theory and research. Journal of Applied Psychology, 102(3), 530-545. http://doi.org/10.1037/apl0000103

James, L. R. (1982). Aggregation bias in estimates of perceptual measures. Journal of Applied Psychology, 67(2), 219-229. http://doi.org/10.1037/0021-9010.67.2.219

James, L. R., Demaree, R. G., \& Wolf, G. (1984). Estimating within-group interrater reliability with and without response bias. Journal of Applied Psychology, 69(1), 85-98. http://doi.org/10.1037/00219010.69.1.85

Kozlowski, S. W. J., \& Klein, K. J. (2000). A multilevel approach to theory and research in organizations: contextual, temporal, and emergent processes. In K. J. Klein \& S. W. J. Kozlowski (Eds.), Multilevel theory, research, and methods in organizations (pp. 3-90). San Francisco: Jossey-Bass.

Kuenzi, M., \& Schminke, M. (2009). Assembling fragments into a lens: A review, critique, and proposed research agenda for the organizational work climate literature. Journal of Management, 35(3), 634-717. https://doi.org/10.1177/0149206308330559

LeBreton, J. M., \& Senter, J. L. (2008). Answers to 20 questions about interrater reliability and interrater agreement. Organizational Research Methods, 11(4), 815-852. https://doi.org/10.1177/1094428106296642

Lindell, M. K., \& Brandt, C. J. (2000) Climate quality and climate consensus as mediators or the relationship between organizational antecedents and outcomes. Journal of Applied Psychology, 85(3), 331-348. http://doi.org/10.1037/0021-9010.85.3.331

Luria, G. (2008). How leaders form consensus. Leadership Quarterly, 19(1), 42-53. https://doi.org/10.1016/j.leaqua.2007.12.004

Meyer, R. D., Dalal, R. S., \& Hermida, R. (2010). A review and synthesis of situational strength in the organizational sciences. Journal of Management, 36(1), 121-140. https://doi.org/10.1177/0149206309349309

Mischel, W. (1973). Toward a cognitive social learning reconceptualization of personality. Psychological Review, 80(4), 252-283. http://doi.org/10.1037/h0035002 
Nyberg, A. J., \& Ployhart, R. E. (2013). Context-emergent turnover (CET) theory: A theory of collective turnover. Academy of Management Review, 38(1), 109-131. http://doi.org/10.5465/amr.2011.0201

Ostroff, C., \& Fulmer, A. (2014). Variance as a construct: Understanding variability beyond the mean. In J. K. Ford, J. R. Hollenbeck, \& A. M. Ryan (Eds.), The nature of work: Advances in psychological theory, methods and practice (pp. 185-210). Washington, DC, US: American Psychological Association. http://doi.org/10.1037/14259-010

Ostroff, C., Kinicki, A. J., \& Muhammed, R S. (2013). Organizational culture and climate. In I. B. Weiner, N. Schmitt, \& S. W. Highhouse (Eds.), Handbook of psychology: Industrial and organizational psychology (pp. 643-676). Hoboken, NJ: Wiley \& Sons. http://doi.org/10.1002/0471264385.wei1222

Park, T., \& Shaw, J. D. (2013). Turnover rates and organizational performance: A meta-analysis. Journal of Applied Psychology, 98(2), 268-309. http://doi.org/10.1037/a0030723

Potočnik, K., Tordera, N. Martínez-Tur, V., Peiró, J. M., \& Ramos, J. (2011). Is service climate strength beneficial or detrimental for service quality delivery? European Journal of Work \& Organizational Psychology, 20(5), 681-699. http://doi.org/10.1080/1359432X.2010.496649

Rafferty, A. E., \& Jimmieson, N. L. (2010). Team change climate: A group-level analysis of the relationships among change information and change participation, role stressors and well-being. European Journal of Work \& Organizational Psychology, 19(5), 551-586. http://doi.org/10.1080/13594320903007869

Rocha, F., Sobrinho, \& Porto, J. B. (2012). Bem-estar no trabalho: Um estudo sobre suas relações com clima social, coping e variáveis demográficas. Revista de Administração Contemporânea, 16(2). Recuperado de http://www.scielo.br/pdf/rac/v16n2/v16n2a06.pdf. http://doi.org/10.1590/S141565552012000200006

Sanders, K., Dorenbosch, L., \& Reuver, R. (2008). The impact of individual and shared employee perception of HRM on affective commitment. Personnel Review, 37(4), 412-425. https://doi.org/10.1108/00483480810877589

Schneider, B. (1975). Organizational climates: An essay. Personnel Psychology, 28(4), 447-479. http://doi.org/10.1111/j.1744-6570.1975.tb01386.x

Schneider, B. (1987). The people make the place. Personnel Psychology, 40(3), 437-453. https://doi.org/10.1111/j.1744-6570.1987.tb00609.x

Schneider, B., Ehrhart, M. G., \& Macey, W. A. (2013). Organizational climate and culture. Annual Review of Psychology, 64, 361-388. https://doi.org/10.1146/annurev-psych-113011-143809

Schneider, B., Goldstein, H. W., \& Smith, D. B. (1995). The ASA framework: An update. Personnel Psychology, 48(4), 747-773. http://doi.org/10.1111/j.1744-6570.1995.tb01780.x

Schneider, B., Gonzáles-Romá, V., Ostroff, C., \& West, M. A. (2017). Organizational climate and culture: Reflections on the history of the constructs in the Journal of Applied Psychology. Journal of Applied Psychology, 102(3), 468-482. http://doi.org/10.1037/ap10000090

Schneider, B., Macey, W. H., Lee, W. C., \& Young, S. A. (2009). Organizational service climate drivers of the American customer satisfaction index (ACSI) and financial and market performance. Journal of Service Research, 12(1), 3-14. https://doi.org/10.1177/1094670509336743

Schneider, B., Salvaggio, A. N., \& Subirats, M. (2002). Climate strength: A new direction for climate research. Journal of Applied Psychology, 87(2), 220-229. http://doi.org/10.1037/00219010.87.2.220 
Schulte, M., Ostroff, C., Shmulyian, S., \& Kinicki, A. (2009). Organizational climate configurations: Relationships to collective attitudes, customer satisfaction, and financial performance. Journal of Appllied Psychology, 94(3), 618-634. http://doi.org/10.1037/a0014365

Shaw, J. D., Gupta, N., \& Delery, J. E. (2005). Alternative conceptualizations of the relationship between voluntary turnover and organizational performance. Academy of Management Journal, 48(1), 50-68. http://doi.org/10.5465/AMJ.2005.15993112

Sowinski, D. R., Fortmann, K. A., \& Lezotte, D. V. (2008). Climate for service and the moderating effects of climate strength on customer satisfaction, voluntary turnover, and profitability. European Journal of Work \& Organizational Psychology, 17(1), 73-88. http://doi.org/10.1080/13594320701473065

Steel, R. P., \& Lounsbury, J. W. (2009). Turnover process models: Review and synthesis of a conceptual literature. Human Resource Management Review, 19(4), 271-282. https://doi.org/10.1016/j.hrmr.2009.04.002

Tsui, A. S., \& O'Reilly, C. A. (1989). Beyond simple demographic effects: The importance of relational demography in superior-subordinate dyads. Academy of Management Journal, 32(2), 402-423. http://doi.org/10.2307/256368

Van Vianen, A. E. M., De Pater, I. E., Bechtoldt, M. E., \& Evers, A. (2011). The strength and quality of climate perceptions. Journal of Managerial Psychology, 26(1), 77-92. https://doi.org/10.1108/02683941111099637

Veloso, E. F. R., Dutra, J. S., \& Nakata, L. E. (2016). Percepção sobre carreiras inteligentes: Diferenças entre as gerações $\mathrm{y}, \mathrm{x}$ e baby boomers. Revista de Gestão, 23(2), 88-98. https://doi.org/10.1016/j.rege.2015.05.001

Zohar, D., \& Luria, G. (2004). Climate as a social-cognitive construction of supervisory safety practices: Scripts as a proxy of behavioral patterns. Journal of Applied Psychology, 89(2), 322-333. http://doi.org/10.1037/0021-9010.89.2.322

\section{Autores}

Tatiana Iwai

Rua Quatá, 300, 04546-042, São Paulo, SP, Brasil.

E-mail: tatianai@insper.edu.br

Adriana Bruscato Bortoluzzo

Rua Quatá, 300, 04546-042, São Paulo, SP, Brasil.

E-mail: adrianab@insper.edu.br

Lina Eiko Nakata

Rua Francisco Leitão, 72, 05414-020, São Paulo, SP, Brasil.

E-mail: linankt@gmail.com, linankt@yahoo.com

José Eduardo Teixeira Costa

Rua Quatá, 300, 04546-042, São Paulo, SP, Brasil.

E-mail: joseeduardo.costa@abril.com.br 